TITLE:

\title{
Features associated with foveal retinal detachment in myopic macular retinoschisis.
}

\section{$\operatorname{AUTHOR}(\mathrm{S}):$}

Fujimoto, Masahiro; Hangai, Masanori; Suda, Kenji; Yoshimura, Nagahisa

\section{CITATION:}

Fujimoto, Masahiro ...[et al]. Features associated with foveal retinal detachment in myopic macular retinoschisis.. American journal of ophthalmology 2010, 150(6): 863-870.e1

\section{ISSUE DATE:}

2010-12

URL:

http://hdl.handle.net/2433/134552

\section{RIGHT:}

(C) 2010 Elsevier Inc:; This is not the published version. Please cite only the published version.; この論文は出版社版でありません。引用の際に は出版社版をご確認ご利用ください。 
Features Associated with Foveal Retinal Detachment in Myopic Macular Retinoschisis

MASAHIRO FUJIMOTO, MASANORI HANGAI, KENJI SUDA, NAGAHISA YOSHIHISA

Department of Ophthalmology and Visual Sciences, Kyoto University Graduate School of Medicine, Japan

Running head: Features in Myopic Macular Retinoschisis

Correspondence to Masanori Hangai, MD, Department of Ophthalmology and Visual Sciences, Kyoto University Graduate School of Medicine, 54 Kawahara-cho, Shogoin, Sakyo-ku, Kyoto 606-8507, Japan

Phone: +81-75-751-3259; Fax: +81-75-752-0933

E-mail: hangai@kuhp.kyoto-u.ac.jp 
Features in Myopic Macular Retinoschisis. Fujimoto et al.--1

\section{INTRODUCTION}

Optical coherence tomography (OCT) has shown that myopic macular retinoschisis (also called foveoschisis) is not uncommon in highly myopic eyes. ${ }^{1,2}$ Retinoschisis was detected in $9 \%$ to $34 \%$ of highly myopic eyes with a posterior staphyloma. ${ }^{1-4}$ Based on the OCT features of untreated and treated eyes, the pathogenesis of myopic macular retinoschisis has been attributed to strong traction on the retina exerted by residual posterior vitreous cortex, an internal limiting membrane (ILM), retinal vessels, or a combination of these. ${ }^{1,5-13}$ Axial length elongation and/or formation of posterior staphyloma in highly myopic eyes may generate the inward tractional force exerted by these factors. This is strongly supported by the remission of the retinoschisis after vitrectomy combined with ILM peeling, which theoretically releases the tractional forces exerted by the posterior vitreous cortex and the ILM and partly by the retinal vessels in the area from which the ILM was peeled. ${ }^{3,14-16}$

Myopic macular retinoschisis progresses to a retinal detachment in $21 \%$ to $43 \%$ of eyes, whereas this disease is often stable for many years without progression to a retinal detachment in other eyes. ${ }^{12,13}$ Development of a foveal retinal detachment in myopic macular retinoschisis impairs vision more severely. Thus, it is clinically important to identify the risk of progression of macular retinoschisis to a foveal retinal detachment. However, it is difficult to determine which eyes with myopic macular retinoschisis develop a retinal detachment. With more recently developed spectral-domain (SD) OCT technology, macular features can be visualized in great detail. ${ }^{17-20}$ Commercially available SD-OCT instruments acquire images 43 to 133 times faster than time-domain (Stratus) OCT instruments. High-speed imaging allows acquisition of high-definition SD-OCT B-scan images ${ }^{20}$ or averaging of multiple OCT B-scans at each location of interest on the retina to reduce speckle noise, thus providing a more detailed view of the macular anatomy. ${ }^{21,22}$ In the current study, we evaluated SD-OCT images enhanced by high-definition or reduction of speckle noise obtained from eyes with myopic foveoschisis to differentiate the morphologic characteristics between eyes with and without a retinal detachment.

\section{METHODS}

Twenty-one highly myopic eyes of 19 patients ( 3 men, 16 women) with macular 
retinoschisis who underwent enhanced SD-OCT examinations were included. In this study, eyes with an axial length equal to or greater than $26.00 \mathrm{~mm}$ were defined as highly myopic.

All patients underwent comprehensive ophthalmologic examinations, including autorefractometry, uncorrected and best-corrected visual acuity measurements using the 5-meter Landolt chart, axial length measurement using the IOL Master (Carl Zeiss Meditec, Dublin, CA), slit-lamp examinations, intraocular pressure measurements using Goldmann applanation tonometry, dilated indirect slit-lamp biomicroscopy, and color fundus photography.

\section{ENHANCED SD-OCT EXAMINATION}

Experienced ophthalmologists examined all eyes using primarily SD-OCT instruments. Enhanced SD-OCT imaging was performed with the Spectralis ${ }^{\mathrm{TM}}$ HRA+OCT system (Heidelberg Engineering, Heidelberg, Germany), RTVue-100 (Optovue, Fremont, CA), and/or Cirrus HD-OCT (Carl Zeiss Meditec) to investigate the retinal microstructures. Enhancement of the B-scan images was achieved by reducing the speckle noise in Spectralis HRA+OCT and RTVue-100 by averaging 12 to 50 multiple OCT B-scans at each precise location of interest on the retina. ${ }^{22}$ Enhancement of the B-scans in Cirrus HD-OCT was achieved by acquisition of high-definition 6-mm B-scans (4,096 axial scans/image) in 0.3 second per image. ${ }^{20}$

\section{RESULTS}

The demographic data from all the patients (16 women, 3 men) are shown in Table 1. The ages of the patients ranged from 37 to 77 years (mean $\pm S D, 65.9 \pm 6.6$ years). The axial lengths ranged from 26.8 to $34.2 \mathrm{~mm}$ (mean \pm SD, $29.7 \pm 2.0 \mathrm{~mm}$ ). Of the 21 eyes, 20 $(95.2 \%)$ eyes had posterior staphyloma.

On OCT images, myopic macular retinoschosis in the outer retina was seen in all eyes. On enhanced SD-OCT images, splitting of the outer retina appeared to be present between the outer plexiform layer and the outer nuclear layer, because in all eyes the highly reflective layer representing the inner plexiform layer and two highly reflective lines representing the external limiting membrane (ELM) and the photoreceptor inner and outer segment junction (IS/OS) were seen in the anterior and posterior borders of the 
retinoschisis, respectively (Figures 1, 2, 3, 4). In 20 (95.2\%) of 21 eyes, multiple columnar structures were seen widely within the retinoschisis as long straight highly reflective lines at the fovea and throughout the retinoschisis. In six eyes in which progression to a foveal retinal detachment was observed during follow-up, the columnar structures within the area of the retinal detachment shortened markedly and lost the columnar shape as if they shrank (Figure 3). In addition to the retinoschisis in the outer retina, there were two other intraretinal separations on the SD-OCT images.

Retinoschisis was found at the level of the inner plexiform layer (IPL; termed IPL schisis in this study) in 11 eyes (50.0\%), and an inner limiting membrane (ILM) detachment was seen in $11(50.0 \%)$ eyes, and both the features were found in nine eyes $(42.9 \%)$. In the space beneath the ILM and within the IPL retinoschisis, multiple columnar structures were observed similar to those seen in the retinoschisis in the outer retina (Figures 1, 2). The ILM detachment was in the superior and/or inferior peripheral macula on vertical B-scans in all 11 eyes, but it was seen in only two eyes on the horizontal B-scans. A comparison with simultaneously obtained infrared images indicated that the retinal vessels were present in the area of the ILM detachments in all eyes (Figures 1, 2). Microfolds were seen in two (9.5\%) of the 21 eyes, which corresponded to a retinal vessel; one was seen within an area with an ILM detachment and IPL retinoschisis (Figure 2) and the other was not associated with these features (Figure 3). A posterior hyaloid membrane was seen in only three eyes (14.3\%) (Figure 4).

A foveal retinal detachment was found at the initial examination in seven $(33.3 \%)$ of the 21 eyes and developed during follow-up in six (28.6\%) eyes. Ultimately, $13(61.9 \%)$ of 21 eyes developed a foveal retinal detachment. Macular holes developed in two of the 21 eyes. Patients who developed a foveal retinal detachment were younger by a mean of 6.4 years, but this difference was not significant (Table 2). There were no significant differences in the percentages of men or women or axial length between patients with and without a foveal retinal detachment (Table 2). Among the features visible on SD-OCT images, only an ILM detachment was found more frequently $(P=0.008)$ in eyes with a foveal retinal detachment $(76.9 \%)$ than eyes without a foveal retinal detachment $(12.5 \%)$ (Table 2).

\section{DISCUSSION}


Enhanced SD-OCT image showed multiple columnar structures within the retinoschisis space that were consistent with previous studies, but these SD-OCT images showed more dense columnar structures in the outer retina throughout the macula compared with previously published single TD-OCT scan images. ${ }^{1,5,23}$ More importantly, this structure was seen not only in the retinoschisis in the outer retina but also in the split spaces beneath the ILM detachment and in the retinoschisis space in the IPL. All columnar structures were long and straight highly reflective lines bridging the inner and outer structures of the retinoschisis spaces, as originally described by Benhamou et al., ${ }^{5}$ suggesting that they are longitudinally stretched; in fact, in the six eyes that progressed to a foveal retinal detachment during follow-up, the columnar structures appeared to shrink and lose the straight shape. These findings suggested that a columnar structure itself has or transmits a tractional force like a spring. Shimada et al. ${ }^{24}$ suggested in a longitudinal study of five eyes with retinoschisis that progressed to a foveal retinal detachment using time-domain OCT that inward traction was transmitted to the outer retina through the foveal columnar structures in the retinoschisis layer. The presence of the dense columnar structures at all the 3 levels of the retinoschisis throughout the macula visible on enhanced SD-OCT images confirmed the presumed involvement of these unique structures in the development of a foveal retinal detachment.

The presence of the columnar structures does not account for why some eyes with myopic macular retinoschisis had or progressed to a foveal retinal detachment, whereas other eyes did not, because in the current study multiple columnar structures were seen in almost all eyes on SD-OCT images in which the speckle noise was reduced. Among the features visible on these SD-OCT images, only an ILM detachment was associated significantly with whether eyes with retinoschisis had or progressed to a foveal retinal detachment. Although myopic macular retinoschisis is sometimes accompanied by an ILM detachment, the relevance of this feature is unclear. ${ }^{13,25}$ Because multiple columnar structures were also present in the intraretinal spaces beneath the ILM detachment, inward traction on the ILM can be transmitted through the columnar structures ultimately to the foveal outer retina. Taken together, an inward tractional force that causes ILM detachment might be transmitted through the intraretinal tissue and columnar structure at each level of the retinoschisis to the foveal photoreceptor layer. Thus, an ILM detachment may be a sign of strong inward traction in the retina that causes a foveal 
Features in Myopic Macular Retinoschisis. Fujimoto et al.--5

retinal detachment.

The cause of the ILM detachment is still uncertain. In the current study, the ILM detachments were always in the peripheral macula where there were retinal vessels including arterioles. A comparison of cross-sectional images with simultaneously obtained intraretinal images indicated that retinal vessels were present in the areas of the ILM detachment. Inward traction generated by retinal vasculature as a result of ocular elongation may cause myopic macular retinoschisis. ${ }^{9,10}$ Ikuno et al. ${ }^{9}$ showed that retinal microfolds commonly form after vitrectomy combined with ILM peeling as a result of residual inward traction exerted by the retinal arterioles in highly myopic eyes. Those authors showed that the locations of the retinal microfolds corresponded to those of the retinal arterioles mainly in the peripheral macula. In the current subjects, the ILM detachment was a primary feature and microfolds were seen only in some eyes before surgery. However, the location of the ILM detachment appears to be similar to that of the microfolds. If retinal vessels have inward traction as a result of ocular elongation, they could exert inward traction directly to the adjacent ILM, which may be partly responsible for the ILM detachments.

Posterior staphyloma may be responsible for the mechanism by which foveal detachment occurs in eyes with macular retinoschisis. In our study, $95.2 \%$ eyes had posterior staphyloma, but it was uncertain whether posterior staphyloma was responsible for the foveal detachment or ILM detachment. Baba et al. ${ }^{2}$ reported that foveal retinal detachment was found in $9.0 \%$ highly myopic eyes with posterior staphyloma but not in highly myopic eyes without posterior staphyloma. Benhamou et al. ${ }^{5}$ suggested that posterior staphyloma is responsible for the development of retinal detachment in highly myopic eyes with a macular hole. This finding was based on long OCT B-scan images showing that in some cases, the retina seemed to be stretched over the staphyloma, similar to the "stretch retinoschisis" described by Green et al. ${ }^{26}$ Green et al. showed that in 1 myopic eye, the peripheral retinal vessels do not lengthen as much as the retina and sclera, thus probably leading to the separation of the retinal vessels and inner limiting membrane from the remainder of the retina. Shimada et al. ${ }^{27}$ showed that the incidence of posterior staphyloma was significantly frequent in eyes with paravascular retinal cysts and that this incidence significantly increased when the vascular lesions included paravascular lamellar holes, which were observed in $83 \%$ of 
the eyes with macular retinoschisis. These studies do not present direct evidence of posterior staphyloma as a cause of foveal detachment; however, it is possible that posterior ectasia of the retinal pigment epithelium and the choroid resulting from posterior staphyloma, sclerotic retinal arterioles, and a firm inflexible ILM that cannot lengthen, may together enhance the inward traction exerted by the retinal arterioles, leading to foveal detachment. ${ }^{1,5,9,27}$ The association of ILM detachment with foveal detachment that was observed in our study appears to support the cooperation of posterior staphyloma and retinal arterioles/ILM in this hypothesis.

The presence of a visible posterior hyaloids membrane was not associated with a foveal retinal detachment possibly because the study was limited in that it included only three eyes in which a posterior hyaloid membrane was visible. Larger studies may be required to confirm whether posterior hyaloid membranes are associated with the development of foveal retinal detachments. Nevertheless, our subjects included a case in which the detachment of a posterior hyaloid membrane gradually progressed from the peripheral macular to the fovea, but the posterior hyaloid membrane remained attached to the fovea without a foveal retinal detachment. Thus, traction by the posterior hyaloid membrane could change in contrast to the persistent traction exerted by the retinal vessels. Another limitation of the current study was that it was not a longitudinal or a cohort study. It is possible that some of eyes currently without a foveal retinal detachment will develop one during a longer follow-up period. Prospective longitudinal studies are needed to confirm our results.

Enhanced SD-OCT images enabled improved visualization of the fine structures associated with macular retinoschisis, such as multiple columnar structures. Our previous study showed that speckle noise reduction by averaging more than 4 SD-OCT B-scans achieved significant improvement in contrast-to-noise ratios and also improved ophthalmologists' ability to distinguish retinal structures. ${ }^{21}$ Srinivasan et al. showed that high-definition high-transverse pixel density OCT images improved visualization of photoreceptor and pigment epithelial morphology, as well as thin intraretinal and epiretinal structures. ${ }^{20}$ In the current study, however, the columnar structures were not clearly visualized in the extrafoveal regions. The oblique position of the extrafoveal regions against the OCT probe light may be responsible for the relatively worse depiction of extrafoveal pathologies; particularly in highly myopic eyes, the extrafoveal region is 
located at a more oblique angle to the probe light when this region is situated further apart from the fovea. In addition, because OCT imaging is based on the patients' visual fixation on the fixation signal within an OCT instrument, small involuntary eye movements probably cause the extrafoveal regions to move to a greater extent than the foveal regions. In speckle noise reduction, the larger movements can cause less precise averaging of multiple B-scans, leading to worse depiction of extrafoveal pathologies. Therefore, ophthalmologists should be aware of this limitation of the use of the current enhanced SD-OCT methods in highly myopic eyes for depicting small pathologies in the extrafoveal regions.

In conclusion, the findings of this study suggested that an ILM detachment in the superior and/or inferior peripheral macula is a sign of persistent strong traction that can cause a foveal retinal detachment, which is likely transmitted to the outer retina through the dense columnar structures in the retinoschisis at each retinal level. This feature is useful for managing highly myopic eyes with macular retinoschisis. 
Features in Myopic Macular Retinoschisis. Fujimoto et al.--8

\section{ACKNOWLEDGMENTS}

Funding/Support: This research was supported in part by a Grant-in-Aid for Scientific Research (20592038) from the Japan Society for the Promotion of Science (JSPS), Tokyo, Japan.

Financial Disclosure: Masanori Hangai is a paid advisory board member for NIDEK CO., LTD., and received consulting fees from Topcon Corporation, and Nagahisa Yoshimura is a paid advisory board member for NIDEK CO., LTD., and is a paid advisory board member for Topcon Corporation. None of the other authors has a conflict of interest to disclose.

Contributions of Authors: Design of the study (MF, MH); Data collection (MF, KS); Analysis and interpretation (MF, KS); Writing of the article (MF, MH); Critical revision of the article (NY); Final approval of the article (MF, MH, KS, NY).

Statement about Conformity with Author Information: All investigations of this study adhered to the tenets of the Declaration of Helsinki. This study was approved by the Institutional Review Board and Ethics Committee of the Kyoto University Graduate School of Medicine. Informed consent was obtained for all patient examinations. 
Features in Myopic Macular Retinoschisis. Fujimoto et al.--9

\section{REFERENCES}

1. Takano M, Kishi S. Foveal retinoschisis and retinal detachment in severely myopic eyes with posterior staphyloma. Am J Ophthalmol 1999;128(4):472-476.

2. Baba T, Ohno-Matsui K, Futagami S, et al. Prevalence and characteristics of foveal retinal detachment without macular hole in high myopia. Am J Ophthalmol 2003;135(3):338-342.

3. Ikuno $\mathrm{Y}$, Sayanagi $\mathrm{K}$, Ohji M, et al. Vitrectomy and internal limiting membrane peeling for myopic foveoschisis. Am J Ophthalmol 2004;137(4):719-724.

4. Panozzo G, Mercanti A. Optical coherence tomography findings in myopic traction maculopathy. Arch Ophthalmol 2004;122(10):1455-1460.

5. Benhamou N, Massin P, Haouchine B, Erginay A, Gaudric A. Macular retinoschisis in highly myopic eyes. Am J Ophthalmol 2002;133(6):794-800.

6. Bando H, Ikuno Y, Choi JS, Tano Y, Yamanaka I, Ishibashi T. Ultrastructure of internal limiting membrane in myopic foveoschisis. Am J Ophthalmol 2005;139(1):197-199.

7. Matsumura N, Ikuno Y, Tano Y. Posterior vitreous detachment and macular hole formation in myopic foveoschisis. Am J Ophthalmol 2004;138(6):1071-1073.

8. Polito A, Lanzetta P, Del Borrello M, Bandello F. Spontaneous resolution of a shallow detachment of the macula in a highly myopic eye. Am J Ophthalmol 2003;135(4):546-547.

9. Ikuno Y, Gomi F, Tano Y. Potent retinal arteriolar traction as a possible cause of myopic foveoschisis. Am J Ophthalmol 2005;139(3):462-467.

10. Sayanagi K, Ikuno Y, Gomi F, Tano Y. Retinal vascular microfolds in highly myopic eyes. Am J Ophthalmol 2005;139(4):658-663.

11. Sayanagi K, Ikuno Y, Tano Y. Reoperation for persistent myopic foveoschisis after primary vitrectomy. Am J Ophthalmol 2006;141(2):414-417.

12. Shimada N, Ohno-Matsui K, Baba T, Futagami S, Tokoro T, Mochizuki M. Natural course of macular retinoschisis in highly myopic eyes without macular hole or retinal detachment. Am J Ophthalmol 2006;142(3):497-500.

13. Gaucher D, Haouchine B, Tadayoni R, et al. Long-term follow-up of high myopic foveoschisis: Natural course and surgical outcome. Am J Ophthalmol 2007;143(3):455-462. 
14. Kobayashi H, Kishi S. Vitreous surgery for highly myopic eyes with foveal detachment and retinoschisis. Ophthalmology 2003;110(9):1702-1707.

15. Kanda S, Uemura A, Sakamoto Y, Kita H. Vitrectomy with internal limiting membrane peeling for macular retinoschisis and retinal detachment without macular hole in highly myopic eyes. Am J Ophthalmol 2003;136(1):177-180.

16. Hirakata A, Hida T. Vitrectomy for myopic posterior retinoschisis or foveal detachment. Jpn J Ophthalmol 2006;50(1):53-61.

17. Schmidt-Erfurth U, Leitgeb RA, Michels S, et al. Three-dimensional ultrahigh-resolution optical coherence tomography of macular diseases. Invest Ophthalmol Vis Sci 2005;46(9):3393-3402.

18. Alam S, Zawadzki RJ, Choi S, et al. Clinical application of rapid serial Fourier-domain optical coherence tomography for macular imaging. Ophthalmology 2006;113(8):1425-1431.

19. Hangai M, Ojima Y, Gotoh N, et al. Three-dimensional imaging of macular holes with high-speed optical coherence tomography. Ophthalmology 2007;114(4):763-773.

20. Srinivasan VJ, Wojtkowski M, Witkin AJ, et al. High-definition and 3-dimensional imaging of macular pathologies with high-speed ultrahigh-resolution optical coherence tomography. Ophthalmology 2006;113(11):2054.e1-14.

21. Sakamoto A, Hangai M, Yoshimura N. Spectral-domain optical coherence tomography with multiple B-scan averaging for enhanced imaging of retinal diseases. Ophthalmology 2008;115(6):1071-1078.

22. Hangai M, Yamamoto M, Sakamoto A, Yoshimura N. Ultrahigh-resolution versus speckle noise-reduction in spectral-domain optical coherence tomography. Opt Express 2009;17(5):4221-4235.

23. Shimada N, Ohno-Matsui K, Yoshida T, Futagami S, Tokoro T, Mochizuki M. Development of macular hole and macular retinoschisis in eyes with myopic choroidal neovascularization. Am J Ophthalmol 2008;145(1):155-161.

24. Shimada N, Ohno-Matsui K, Yoshida T, Sugamoto Y, Tokoro T, Mochizuki M. Progression from macular retinoschisis to retinal detachment in highly myopic eyes is associated with outer lamellar hole formation. Br J Ophthalmol 2008;92(6):762-764.

25. Sayanagi K, Ikuno Y, Soga K, Tano Y. Photoreceptor inner and outer segment defects in myopic foveoschisis. Am J Ophthalmol 2008;145(5):902-908. 
Features in Myopic Macular Retinoschisis. Fujimoto et al.--11

26. Green WR. Retina, myopia. In: Spencer WH, editor. Ophthalmic pathology: an atlas and textbook. Vol 11. 4th ed. Philadelphia: WB Saunders, 1996:971-972.

27. Shimada N, Ohno-Matsui K, Nishimuta A, et al. Detection of paravascular lamellar holes and other paravascular abnormalities by optical coherence tomography in eyes with high myopia. Ophthalmology 2008;115(4):708-717. 
Features in Myopic Macular Retinoschisis. Fujimoto et al.--12

\section{FIGURE CAPTIONS}

Figure 1. Representative images show an internal limiting membrane (ILM) detachment in the inferior peripheral macula in an eye with a foveal retinal detachment (patient 1). The best-corrected visual acuity is 0.2 . (Top left, and Middle left) Infrared fundus photographs show the green scan lines of the enhanced spectral-domain optical coherence tomography (SD-OCT, Spectralis HRA+OCT) images in (Top, and Middle right), respectively. The red and blue arrows indicate the points at which the scan line crosses the retinal vessels, which correspond to the red and blue arrowheads, respectively, in (Top right). (Top, and Middle right) Vertical and horizontal 9-mm enhanced SD-OCT B-scans show macular retinoschisis and a foveal retinal detachment. (Top right) The ILM detachment (white arrows) and inner nuclear layer schisis are seen between the red arrowheads in the inferior peripheral macula along the vertical scan. The superior point (blue arrow) corresponding to a retinal arteriole does not show any features such as a retinal microfold or ILM detachment. Multiple columnar structures are seen in the hyporeflective spaces within the ILM detachment and outer retinoschisis. (Bottom) The magnified (2X) view of the area outlined by red dashed lines in the SD-OCT images in (Middle right). A hyporeflective space with multiple columnar structures is present between the highly reflective outer plexiform layer (OPL) and highly reflective lines representing the external limiting membrane (ELM) and the photoreceptor inner and outer segment layer junction (IS/OS).

Figure 2. Representative images show an internal limiting membrane (ILM) detachment in the inferior and superior peripheral macula in an eye with foveal retinal detachment (patient 11). The best-corrected visual acuity is 0.1 . (Top left) Color fundus photograph. (Middle left) An infrared fundus photograph shows the red scan lines $\left({ }^{*}, \dagger\right.$, and $\left.\S\right)$. (Top, Middle, and Bottom center) Vertical ( ${ }^{*}$ and $\dagger$ ) and horizontal (§) 6-mm enhanced spectral-domain optical coherence tomography (SD-OCT, Cirrus HD-OCT) images. (Top, Middle, and Bottom right) The magnified (2X) views of the areas outlined by red dashed lines in the SD-OCT images in (Top, Middle, and Bottom center), respectively. The red and blue arrows (Middle left) indicate the points at which the scan line crosses the retinal vessels, which are indicated by the red and blue arrowheads (Top, and Middle center, and Middle right), respectively, in the vertical SD-OCT images. The 
vertical enhanced SD-OCT B-scans show macular retinoschisis and a foveal retinal detachment. The ILM detachment (white arrows) and inner plexiform layer (IPL) schisis are seen in areas including the retinal microfold at the point at which the scan crosses the retinal vessels as shown by the red arrowhead in the inferior peripheral macula along a vertical scan. The crossing point indicated by the blue arrowhead does not show a retinal microfold. IPL schisis is also seen in the superior macula peripheral to the crossing point. Multiple columnar structures are evident in the hyporeflective spaces within the ILM detachment, IPL retinoschisis, and outer retinoschisis. A hyporeflective space with multiple columnar structures is present between the highly reflective outer plexiform layer (OPL) and highly reflective lines representing the external limiting membrane (ELM) and the photoreceptor inner and outer segment layer junction (IS/OS).

Figure 3. Images of an eye that progressed to a foveal retinal detachment without an internal limiting membrane (ILM) detachment (patient 2). This is an exceptional case in which a foveal retinal detachment developed, but no ILM detachment was found at any time during the progression. The images from this case are shown because the entire process of foveal retinal detachment formation is well documented. (Top left) A color fundus photograph shows the scan lines (white lines) in (Second, Third, Fourth, and Fifth row left). (Top center) An infrared fundus photograph shows multiple punctuate lesions corresponding to the columnar structures in the outer retinoschisis. (Top right) An infrared fundus photograph shows the green scan lines in (Sixth row and Bottom left). (Second, Fourth, Fifth, and Sixth row left) Horizontal enhanced spectral-domain optical coherence tomography images. (Third row, and Bottom left) Vertical enhanced SD-OCT. (Second to Fifth row left) 6 mm length B-scans in RTvue-100; (Sixth row, and Bottom left) $9 \mathrm{~mm}$ length B-scans in Spectralis HRA+OCT. (Second, Third, Fourth, Fifth, and Sixth row right) Magnified (2X) views of the areas outlined by red dashed lines on SD-OCT images in (Second, Third, Fourth, Fifth, and Sixth row left), respectively. The OCT images were obtained at the initial visit (Second, and Third row), and 1 month (Fourth row), 2 months (Fifth row), and 5 months (Six row and Bottom) after the initial visit. At the initial visit, the foveal photoreceptor layer appears intact based on the visibility of the highly reflective lines representing the external limiting membrane (ELM) and the photoreceptor inner and outer segment layer junction (IS/OS). At 1 month, 
a small area in the temporal juxtafovea shows a retinal detachment. At 2 months, the retinal detachment includes the whole fovea. At 5 months, the foveal detachment has enlarged, and consequent disruption of the outer photoreceptor layer is seen. The best-corrected visual acuity was $0.6,0.6,0.3$, and 0.3 at the initial visit and at 1 month, 2 months, and 5 months after the initial visit, respectively. During progression to a foveal retinal detachment, the columnar structures indicated by white arrows gradually shrank and did not resemble a column. (Top left, and right) The red arrows point to where the scan lines cross the retinal vessels, indicated by red arrowheads in the vertical SD-OCT images (Third row, and Bottom). No ILM detachment or microfolds are seen.

Figure 4. Images from an eye that did not progress to a foveal retinal detachment for many years without internal limiting membrane detachment (patient 14). (Top left) A color fundus photograph shows the scan lines (white lines) of optical coherence tomography (OCT) images in (Top, Second, Third, and Fourth row right). (Top, Second, and Third row right) Time-domain (TD) OCT (Stratus OCT) B-scan images at the same horizontal scan. (Top right) The initial visit; (Second row right) 6 months after the initial visit; (Third right) 26 months after the initial visit. The white arrows indicate the central edge of the posterior hyaloid membrane detachment. The red arrows indicate the peripheral ends of the retinoschisis in the outer retina. As the posterior hyaloid membrane detachment progressed, the area of the retinoschisis narrowed, and the height of the foveoschisis appeared to increase, but no foveal detachment developed. (Second row left, and Fourth row right) Spectral-domain OCT (SD-OCT, Cirrus OCT) B-scans at the same location and time as the TD-OCT image in (Third row right). (Second row left) A magnified (2X) view of the area outlined by red dashed lines in the SD-OCT images in (Fourth row right). The highly reflective lines representing the external limiting membrane (ELM) and the photoreceptor inner and outer segment layer junction (IS/OS) is nearly intact. (Bottom left) An infrared image simultaneously obtained with SD-OCT images in (Fifth row, and Bottom right) Horizontal (Fifth row right) and vertical (Bottom right) 9-mm length SD-OCT B-scan images (Spectralis HRA+OCT) obtained 33 months after the initial visit. The best-corrected visual acuity was stable during the 33 months $(0.7,0.7,0.6$, and 0.7 at the initial visit and at 6,26 , and 33 months after the initial visit, respectively). The red and blue arrows (Bottom left) indicate the 
Features in Myopic Macular Retinoschisis. Fujimoto et al.--15

points where the scan lines cross the vessels, indicated by the red and blue arrowheads, respectively (Bottom right). There is a microfold (red arrowhead) at a point corresponding to the retinal vessel but no ILM detachment. 


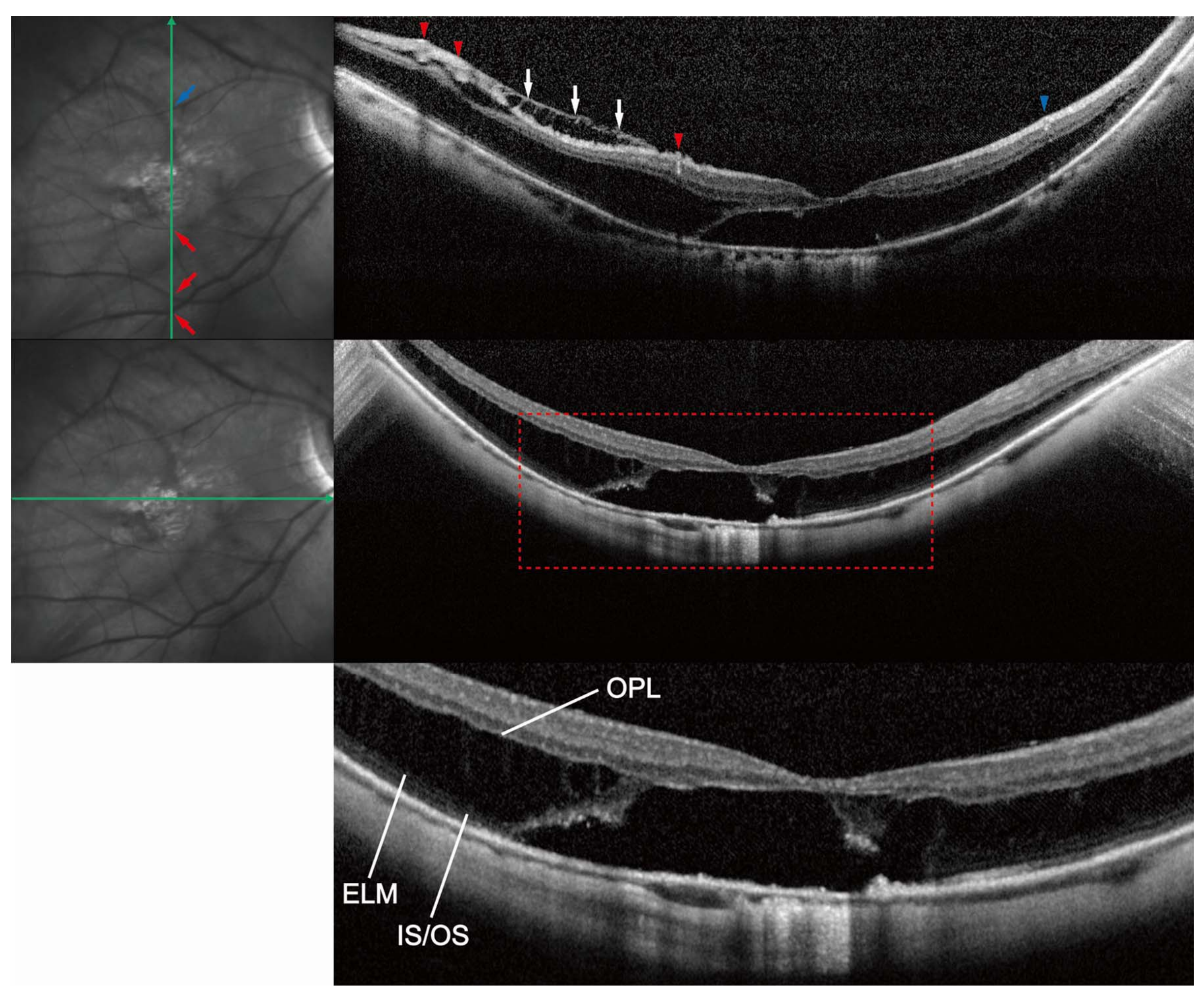




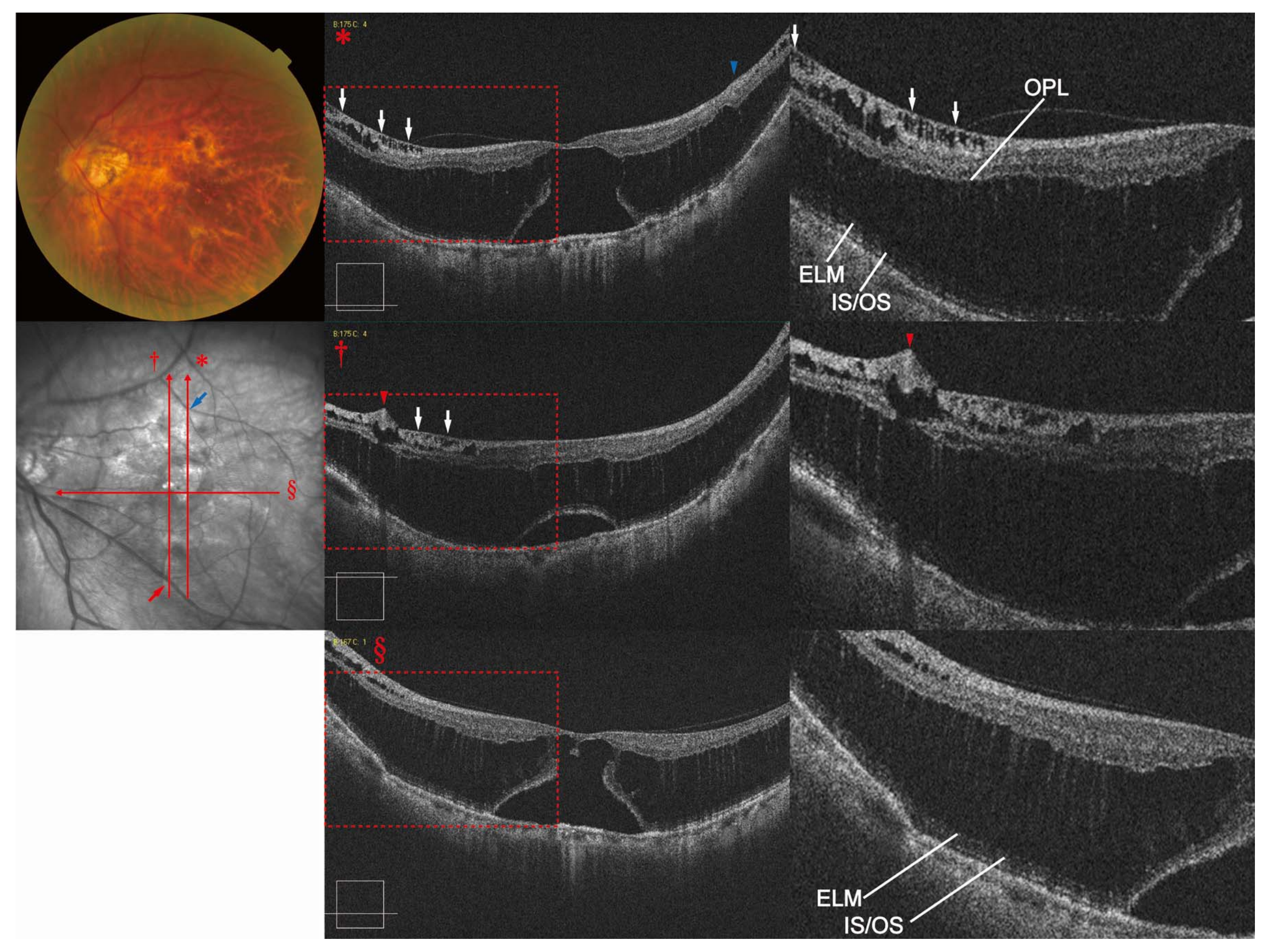



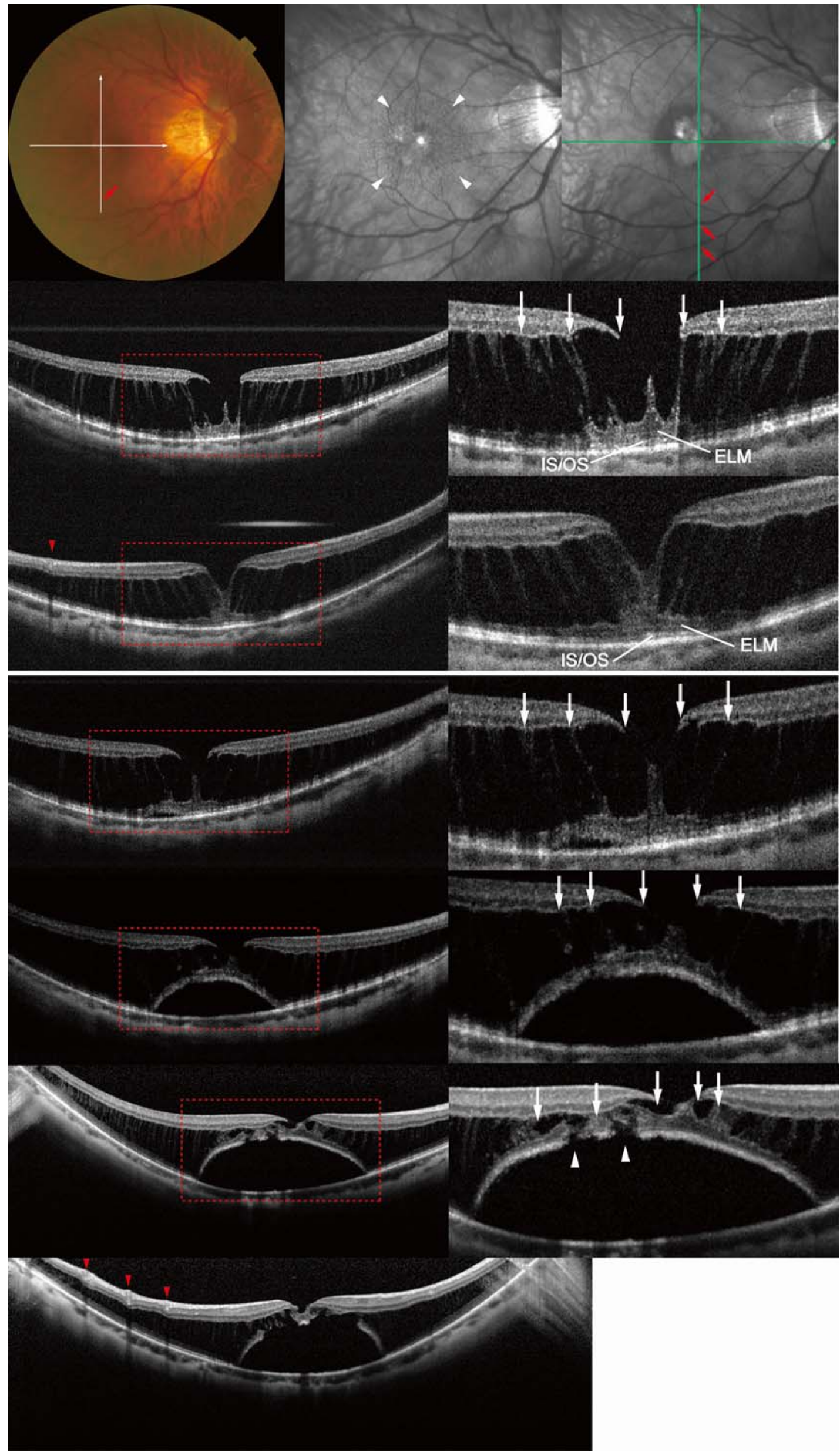


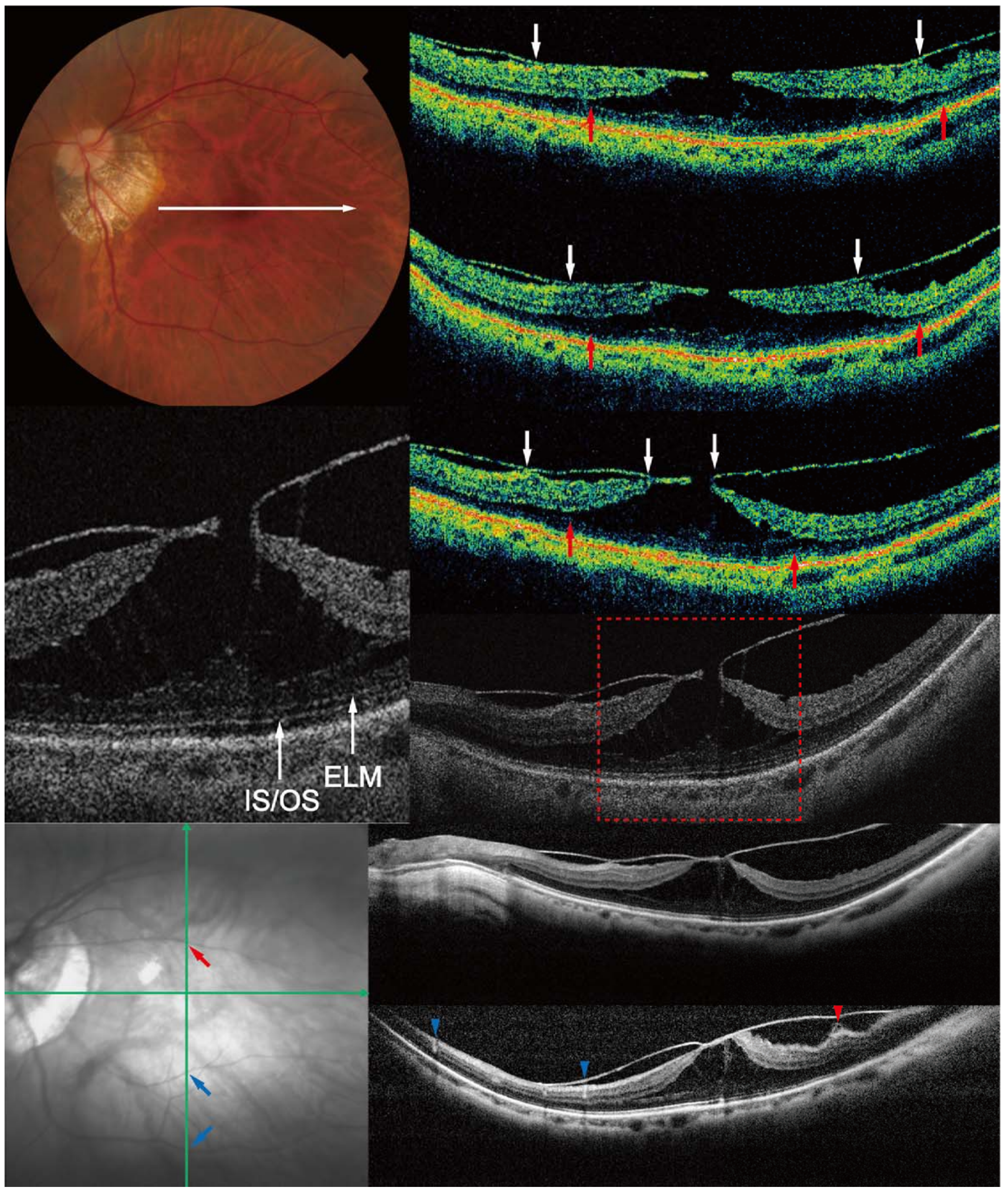

